\title{
CROSSING OVER IN THE MALE MOUSE AS ANALYSED BY RECOMBINATION NODULES AND BARS
}

by

\author{
JOAKIM GLAMANN
}

Department of Physiology, Carlsberg Laboratory

Gamle Carlsberg Vej 10, DK-2500 Copenhagen Valby

Keywords: Meiosis, synaptonemal complex, pachytene, XY bivalent

\begin{abstract}
The present study of 26 serially sectioned mouse spermatocyte nuclei has permitted the following observations and conclusions: 1) The pachytene stage can be subdivided into an early, a mid, and a late substage. 2) A morphological change of the early spheric recombination nodule to an elongated bar is observed. 3) The number of recombination structures per nucleusdecreases from a mean of 31 nodules and 2 bars at early pachytene to a mean of 8 nodules and 6 bars at mid pachytene. The number of nodules and bars remains virtually constant at late pachytene and early diplotene. 4) The number of bivalents with one and two nodules is higher than would be expected if the nodules were distributed at random. 5) Along the bivalents the frequency of nodules and bars exhibits a characteristic pattern of troughs and peaks. 6) The presence of a nodule/bar distally in the synaptonemal complex segment combining the $\mathrm{X}$ and the $\mathrm{Y}$ chromosomes strongly indicates the existence of an obligatory crossover between the terminal parts of the long arm of the $\mathrm{X}$ and $\mathrm{Y}$ chromosomes.
\end{abstract}

\section{INTRODUCTION}

Correspondence between the number and location of recombination nodules and meiotic exchange has been documented in a number of species, suggesting that crossing over is mediated by these electron dense spheres (see 39 for review). This has provided an opportunity to determine the number of crossovers at pachytene in a range of organisms where genetic analyses are scarce $(3,16,17,18,19,40)$. This information can for example be used to compare the number and distribution of crossovers among and along bivalents in males and females of the same species as has been done in human spermatocytes and oocytes $(3,18)$.

Studies of phylogenetically diverse organisms such as fungi and man have shown that an excess of nodules are distributed at random among the bivalents during zygotene and early pachytene. Subsequent replacement of recombination nod- ules leads to numerical as well as distributional changes by the end of pachytene, providing each bivalent with at least one crossover, a prerequisite for a regular chromosome disjunction. These observations are in agreement with a large amount of evidence showing that the number and distribution of crossovers are genetically controlled (1). In Drosophila, meiotic mutations which reduce the frequency of crossing over are easily obtained. These are so-called precondition mutants, in which the number and distribution of the residual crossovers are changed relative to that observed in wild type. An analysis of Drosophila pachytene oocytes from the precondition mutants mei-41 and mei-218 thus showed a reduction in the number of nodules and a change in their distribution which corresponded to the number and distribution of crossovers determined genetically (4).

The mouse is the best genetically character- 
ized mammal. However, in spite of a number of detailed investigations of the ultrastructure of meiosis $(6,7,8,12,25,26)$, in particular the behavior of the $X$ and $Y$ chromosomes $(36,37$, 38 ), and the pairing behavior of rearranged chromosomes $(24,28,29)$, very little is known about the recombination nodules. The analysis described below was therefore initiated with the specific aim of investigating the pachytene stage of meiosis in mouse spermatocytes in order to determine the structural, numerical and distributional changes of recombination nodules as well as to estimate the total number and distribution of crossovers. These results are compared with those available for other organisms $(3,4,16$, $18,19,30,40)$.

\section{MATERIALS AND METHODS}

Male mice of the strain CBA/j obtained from Bomholtgaard (Jutland, Denmark) were sacri- ficed by cervical dislocation when 6,7,20 and 35 weeks old. The testes were swiftly removed through a median abdominal incision.

The initial fixation was in $4 \%$ glutaraldehyde

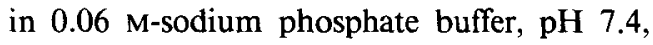
followed by postfixation for 2 hours in $2 \% \mathrm{OsO}_{4}$ in the same buffer. The tissue was en bloc stained in $2 \%$ uranyl acetate for 3 hours at $60{ }^{\circ} \mathrm{C}$, dehydrated in ethanol and embedded in Spurr's resin. The procedures used for fixation, embedding, serial sectioning, and reconstruction of the synaptonemal complexes as well as the methods for measuring the synaptonemal complex lengths have been described in detail elsewhere $(15,30)$.

The general histology of the testis was examined by phase contrast microscopy of $2.5 \mu \mathrm{m}$ thick sections of the material fixed and embedded for electron microscopy. The testes of all sacrificed mice appeared to be normal and contained all stages of spermatogenesis up to

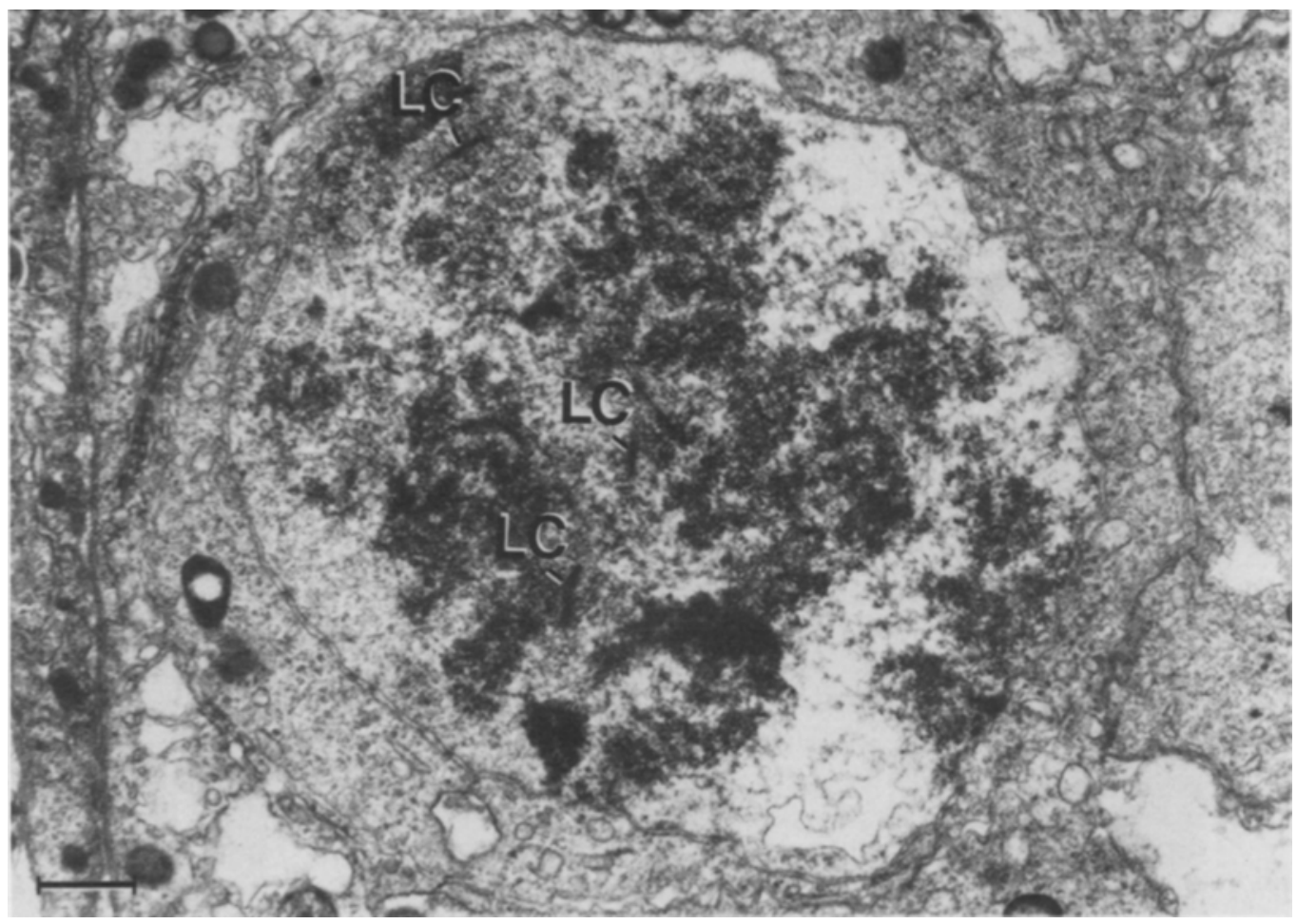

Figure 1. A survey micrograph of a late zygotene nucleus. $\mathrm{LC}$, lateral component. $(\mathrm{Bar}=0,5 \mu \mathrm{m})$. 


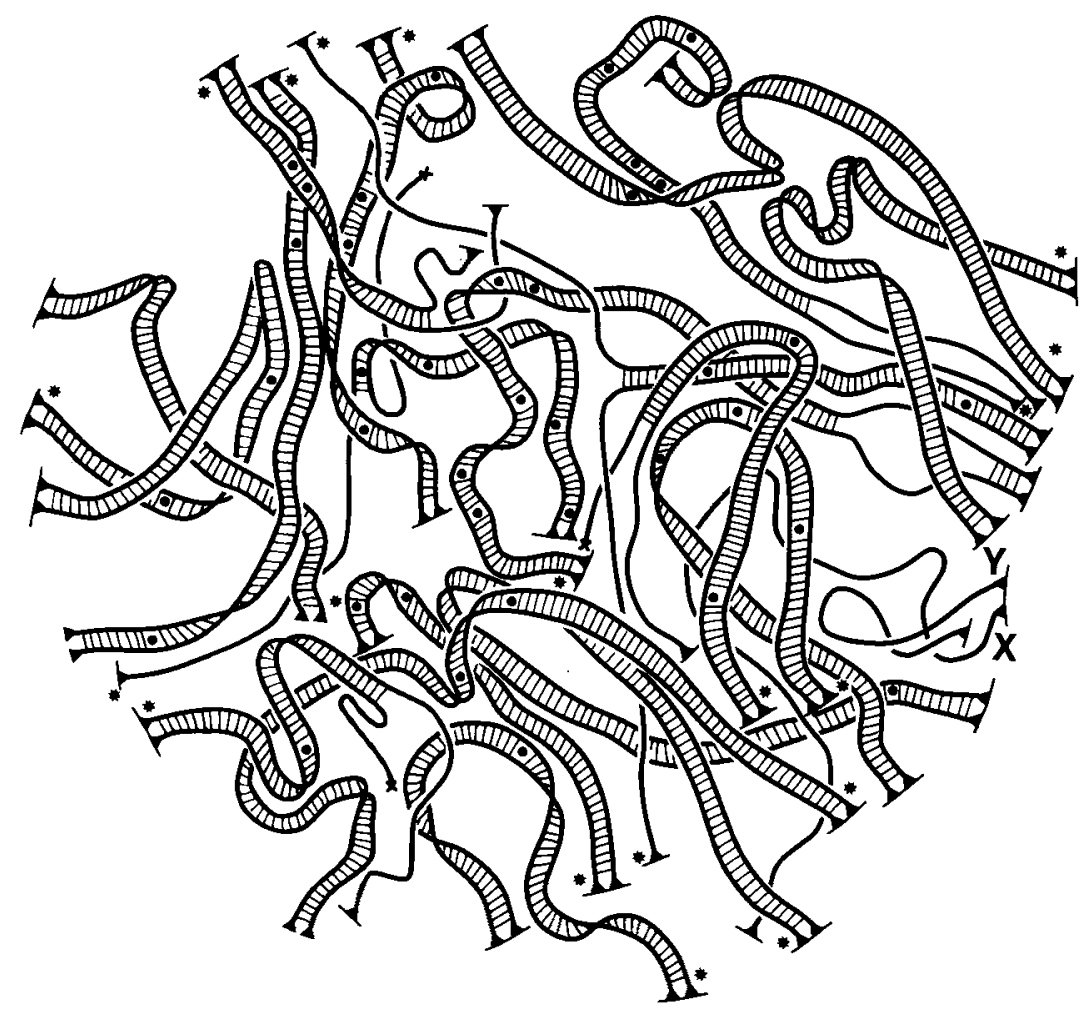

Figure 2. A reconstruction of a late zygotene nucleus. The centromeric heterochromatin is omitted for the sake of clarity and the telomeres of the short arms are denoted by asterisks. Recombination nodules are shown as dots in the central region of the synaptonemal complex and bars as dense lines bridging the central region. $X$ and $Y$, the lateral components of the $\mathrm{X}$ and $\mathrm{Y}$ chromosomes.

mature spermatozoa. Possible age-related structural differences of the seminiferous tubules could not be detected.

Nuclei at the different stages of meiotic prophase could with some experience easily be identified by nuclear size and organization as outlined in the following sections. Following an identification of a cluster of cells in the stage of interest, this region of the seminiferous tubule was serially sectioned and photographed in a Siemens Elmiscope 102.

\section{RESULTS}

\subsection{Stages of meiotic prophase}

A total of 26 nuclei, comprising one late zygotene nucleus, 24 pachytene nuclei, and one early diplotene nucleus, has been serially sectioned and reconstructed. The late zygotene nucleus can be distinguished from the pachytene nuclei by the incomplete synapsis of the autosomes (Figures 1 and 2) and the early diplotene nucleus by the initiation of synaptonemal complex degradation (Figures 7 and 8). The 24 pachytene nuclei reveal a continuous series of changes in nuclear architecture bridging the differences between the late zygotene nucleus and the early diplotene nucleus indicating that the 24 nuclei constitute a representative sample covering the entire pachytene stage of mouse spermatogenesis. The pachytene stage can be divided into an early, a mid, and a late substage represented by 11, 6 and 7 nuclei on the basis of the following characteristics:

Early pachytene: 1) Chromosome bouquet is present in some of the nuclei. 2) The chromatin of the autosomes is condensed. 3) The $X$ and $Y$ chromosomes are extensively synapsed. The XY 
TABLE I. Total mean complement length (half the total lateral component length) and mean number of recombination nodules and bars in mouse spermatocytes. (n, number of nuclei investigated. sd, standard deviation).

\begin{tabular}{lrllll}
\hline Stage & $\mathrm{n}$ & $\begin{array}{l}\text { Complement } \\
\text { length } \pm \mathrm{sd} \\
(\mu \mathrm{m})\end{array}$ & $\begin{array}{l}\text { Number of } \\
\text { recombination } \\
\text { nodules }\end{array}$ & $\begin{array}{l}\text { Number of } \\
\text { recombination } \\
\text { bars }\end{array}$ & $\begin{array}{l}\text { Total number } \\
\text { of recombination } \\
\text { structures }( \pm \mathrm{sd})\end{array}$ \\
\hline Late zygotene & 1 & 112 & 41 & 0 & 41 \\
Early pachytene & 11 & $118 \pm 6$ & 31 & 2.3 & $33 \pm 8$ \\
Mid pachytene & 6 & $104 \pm 12$ & 8 & 6.2 & $14 \pm 3$ \\
Late pachytene & 7 & $159 \pm 8$ & 7 & 7.4 & $14 \pm 6$ \\
Early diplotene & 1 & 156 & 7 & 6 & 13 \\
\hline
\end{tabular}

body is only attached to the nuclear envelope at the sites of telomere attachment.

Mid pachytene: 1) A bouquet is no longer present. 2) The autosomal chromatin is less condensed than at early pachytene. 3 ) The $X$ and $Y$ chromosomes are only paired over a short distance. The XY bivalent starts to flatten out along the nuclear envelope.
Late pachytene: 1) The complement length has increased $50 \%$ and the diameter of the nucleus has increased. 2) The autosomal chromatin is diffuse. 3) The $\mathrm{X}$ and $\mathrm{Y}$ chromosomes are paired only over a short distance. The $X Y$ bivalent is flattened along the nuclear envelope and the unpaired lateral components often reveal longitudinal splitting into two subunits.

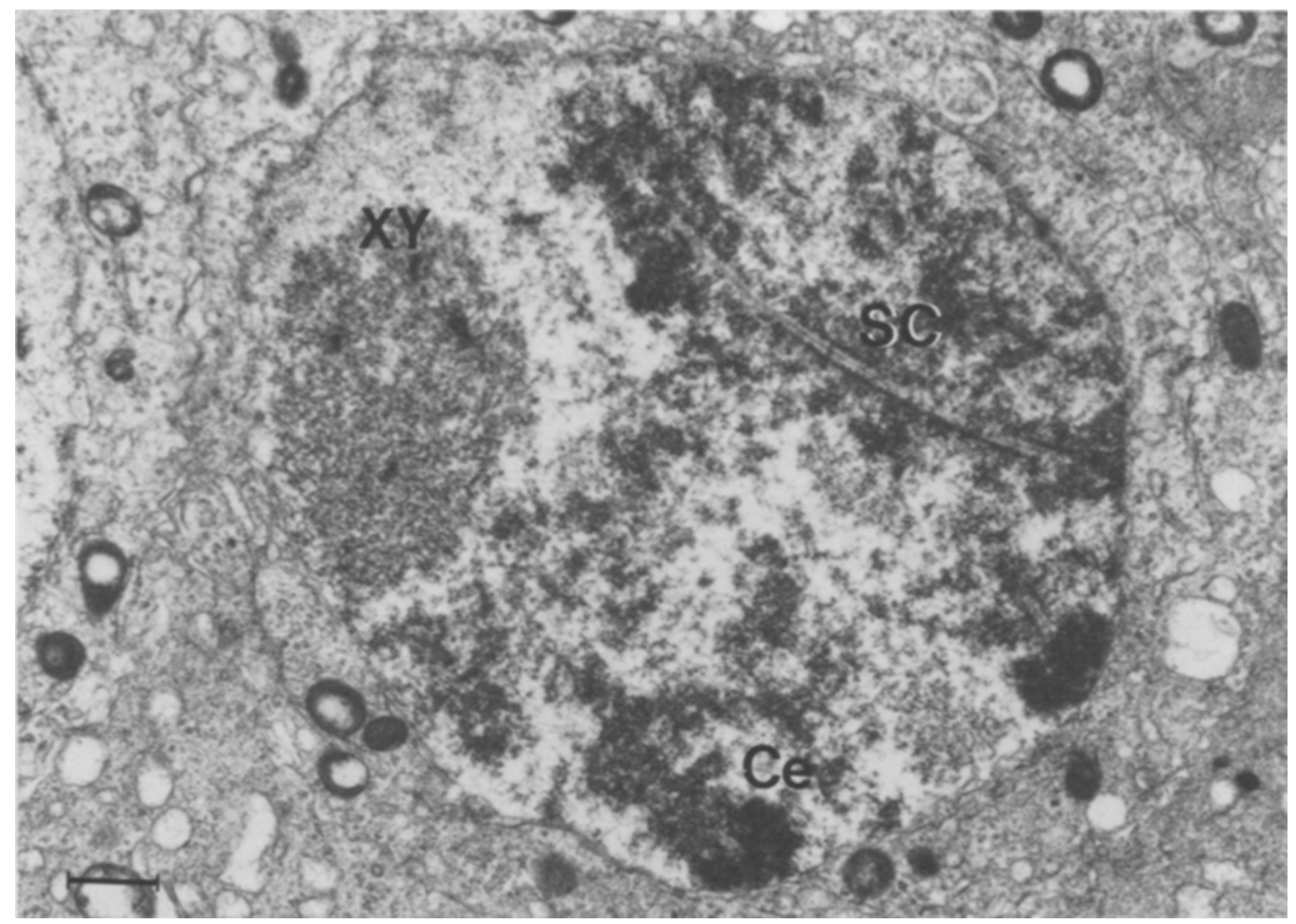

Figure 3. A survey micrograph of an early pachytene nucleus. SC, synaptonemal complex. Ce, centromeric heterochromatin, $\mathrm{XY}, \mathrm{XY}$ bivalent, $(\mathrm{Bar}=0,5 \mu \mathrm{m})$. 


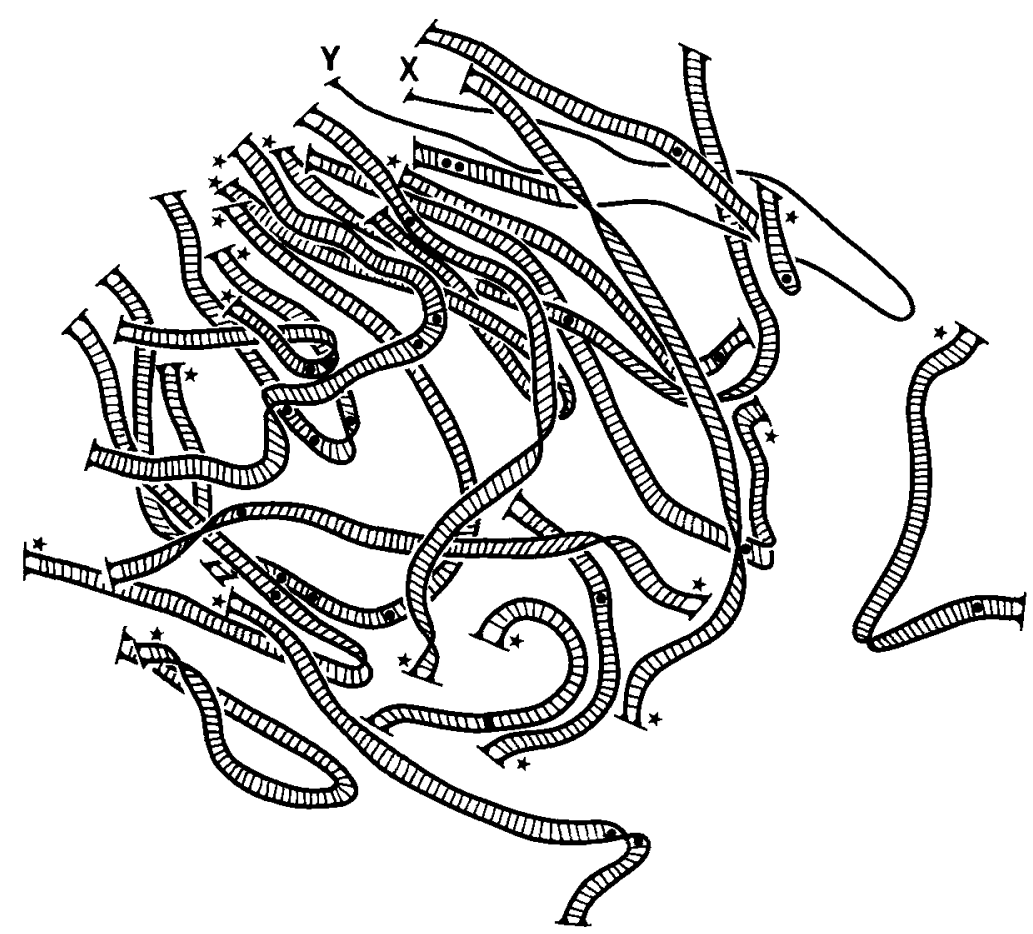

Figure 4. A reconstruction of an early pachytene nucleus. Note the prominent bouquet. An explanation to the symbols is given in the legend to Figure 2.

\subsection{The pachytene nucleus}

\subsubsection{The synaptonemal complex complement}

All reconstructed pachytene nuclei and the reconstructed early diplotene nucleus contain 19 autosomal chromosome pairs and an XY pair and no structural aberrations are seen (Figures 4, 6 and 8 ) with the exceptions mentioned in the following paragraph. In all nuclei the telomeres are attached to the inner membrane of the nuclear envelope.

In the late zygotene nucleus it is only possible to identify 18 partially paired autosomal bivalents (Figure 2), but it is uncertain whether this reflects a numerical aberration or an error in the reconstruction. A ring bivalent (Figure 10) is found in one of the late pachytene nuclei. The ring bivalent lacks normal telomeres though it edges the nuclear envelope with a local thickening of the lateral component at the point of contact. Other reconstructed pachytene nuclei from the same mouse did not contain a ring bivalent indicating that the aberration has origi- nated in the germline of this mouse. The fact that both homologues appear to be similar affected may suggest that the ring has formed at a stage where the two homologues were completely synapsed.

A distinct bouquet configuration caused by the confinement of the telomere attachment sites to a limited region of the nuclear envelope is only observed in two early pachytene nuclei (Figure 2). This is in contrast to the regular presence of a chromosome bouquet at zygotene and early pachytene in most other organisms ( 2 , 17) including the female mouse (35). If bouquet formation is a regular feature of chromosome pairing in mouse spermatocytes, it appears in most nuclei to be resolved before the late $z y-$ gotene-early pachytene stages.

The mean total length of the synaptonemal complex complement at the different stages is given in Table I. It is apparent that the lateral component length remains constant from late zygotene to mid pachytene, whereafter it in- 


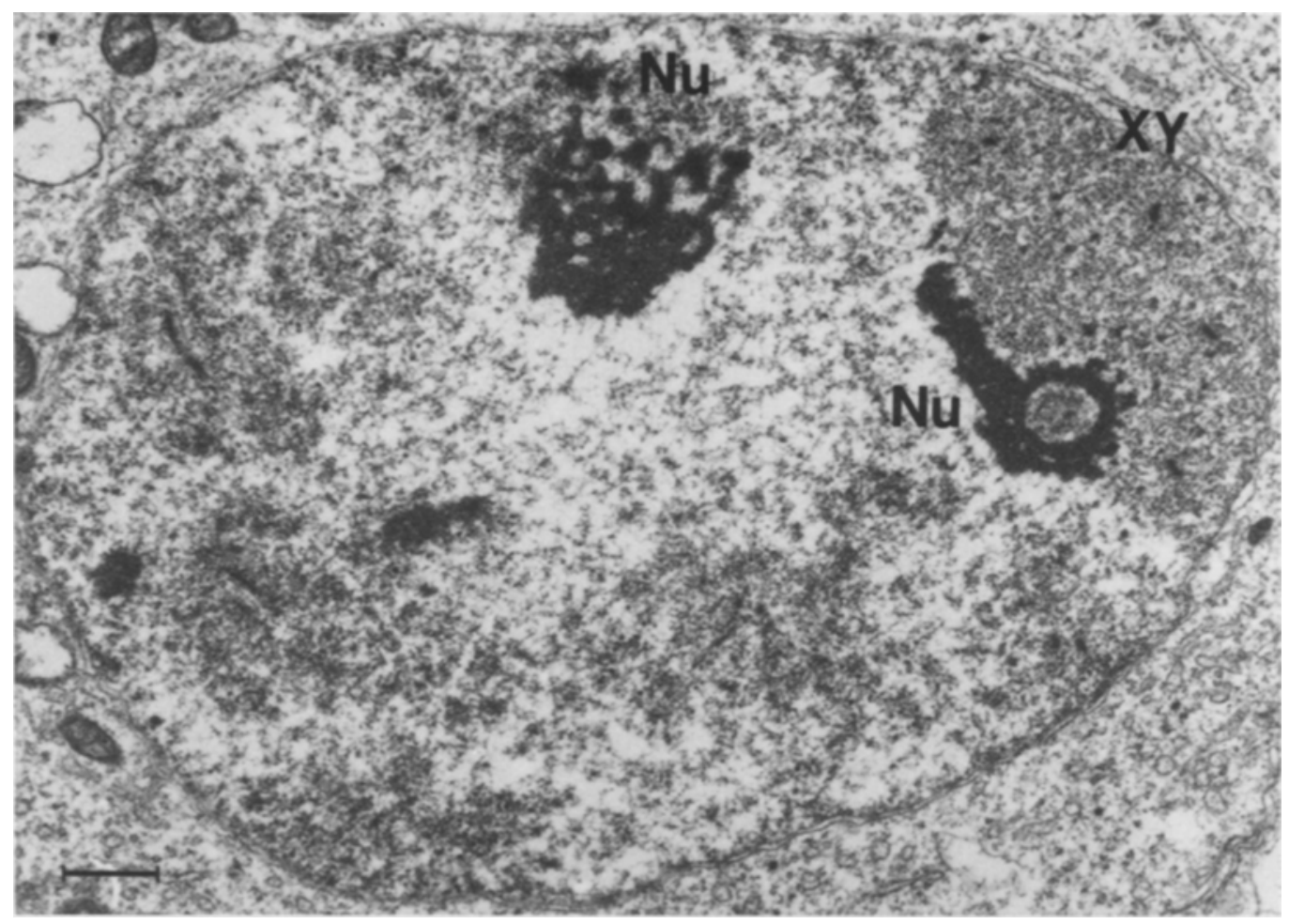

Figure 5. A survey micrograph of a mid pachytene nucleus. $X Y, X Y$ bivalent. Nu, nucleolus. $(B a r=0,5 \mu \mathrm{m})$.

creases by approximately $50 \%$ between mid and late pachytene. The transition from mid to late pachytene is also characterized by an increase in nuclear volume. In general, 80-90 ultrathin sections (0.08 $\mu \mathrm{m}$ in thickness) are needed to encompass the roughly spherical early or mid pachytene nucleus, whereas late pachytene nuclei extend over 105-115 sections.

\subsubsection{The chromatin}

As illustrated by Figures 1, 3, 5, and 7 the organization and electron density of the chromatin gradually change during prophase. At late zygotene the chromatin appears to be condensed around the lateral components and synaptonemal complexes, whereas at late pachytene and early diplotene a more loose configuration is apparent. It is, however, often difficult to use the chromatin condensation as a parameter for subdividing the pachytene stage since the ap- pearance of the chromatin is influenced by even small differences in section thickness and in the processing of the negatives.

All the autosomal bivalents possess a region of condensed centromeric heterochromatin (Figure 9). In general the telomeric region itself is surrounded by less condensed chromatin indicating the existence of a short arm. It is not possible to identify prokinetochores in the centromeric heterochromatin in contrast to the situation in pachytene nuclei of human spermatocytes (17). The centromeric heterochromatin appears unchanged in conformation and density from late zygotene to early diplotene (Figures 1, 3,5 and 7) unlike the situation in human spermatocytes where these regions increase in size and density from leptotene to diplotene. Often the centromeric heterochromatin of several bivalents in the mouse has fused into larger aggregates. 


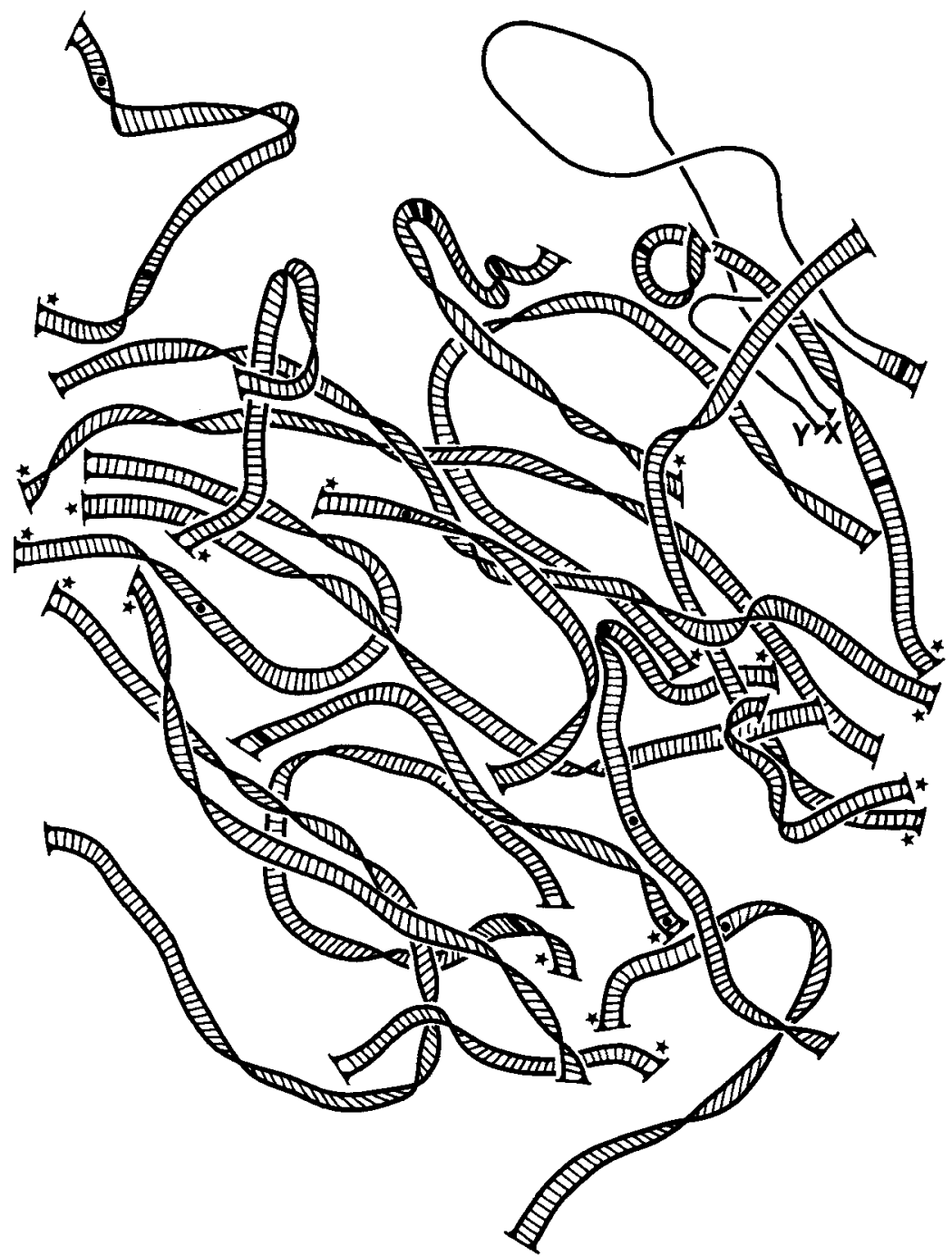

Figure 6. A reconstruction of a mid pachytene nucleus. An explanation to the symbols is given in the legend to Figure 2.

\subsubsection{The XY bivalent}

At late zygotene the $\mathrm{X}$ and $\mathrm{Y}$ chromosomes are indistinguishable from the autosomes, possessing continuous lateral components surrounded by chromatin (Figures 2 and 11 ). Both ends of the chromosomes are attached to the inner membrane of the nuclear envelope and the centromeric heterochromatin at one telomere region can be recognized at late zygotene as discrete regions of more condensed chromatin.
In the reconstructed late zygotene nucleus and in two early pachytene nuclei (100\% pairing of the autosomes) the $\mathrm{X}$ and $\mathrm{Y}$ chromosomes are not paired with a synaptonemal complex.

At early pachytene, the $\mathrm{X}$ and $\mathrm{Y}$ chromosomes have paired and formed a synaptonemal complex between the noncentromeric ends in nine of the eleven nuclei (Table II). The length of the synaptonemal complex combining $X$ and $Y$ varies considerably among the nuclei and can 


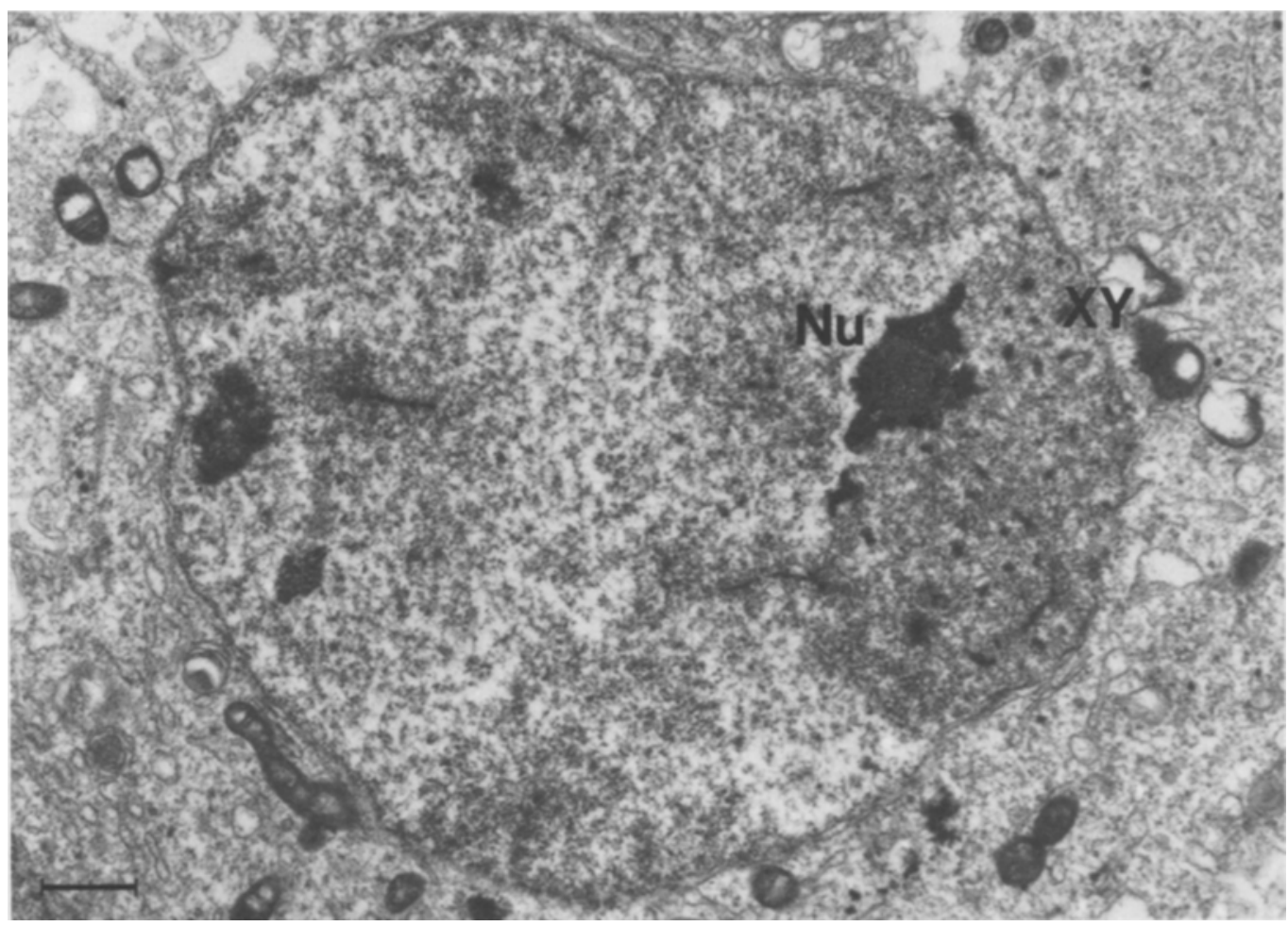

Figure 7. A survey micrograph of an early diplotene nucleus. $\mathrm{XY}, \mathrm{XY}$ bivalent. Nu, nucleolar material associated with the $\mathrm{XY}$ bivalent. (Bar $=0,5 \mu \mathrm{m})$.

reach $55 \%$ of the length of the $\mathrm{Y}$ chromosome. At this stage, the lateral components become embedded in an oval chromatin mass of intermediate density the so-called XY body. The XY body is associated with the nuclear envelope through the attachment sites of the telomeres, and protrudes into the nucleoplasm (Figures 4 and 11). The formation of the $X Y$ body is accompanied by an increase in thickness of the lateral components of the $\mathrm{X}$ and $\mathrm{Y}$ chromosomes.

As pachytene progresses, the length of the synaptonemal complex between the $X$ and $Y$ chromosomes decreases, and at mid and late pachytene only a short piece remains (Table II), In one mid and one late pachytene nucleus the $\mathrm{XY}$ bivalents appear to be devoid of a synaptonemal complex although the two non-centromeric telomeres of the sex chromosomes are closely associated. In seven of the mid and late pachytene nuclei the centromeric telomeres of the sex chromosomes are attached to the nuclear envelope in the vicinity of each other. At mid pachytene the $X Y$ body acquires a finer and more homogeneous appearance (Figure 5) which is preserved at late pachytene where the $\mathrm{XY}$ body is flattened out and appressed to the nuclear envelope (Figure 11). The unpaired lateral component of the $\mathrm{X}$ chromosome frequently reveals local splitting into two subunits, often interspersed with central region-like material. The splitting of the lateral components as well as the flattening of the XY body becomes more extensive at early diplotene. In the reconstructed nucleus the non-centromeric ends of the $\mathrm{X}$ and $\mathrm{Y}$ chromosomes are closely associated as are the centromeric ends, but a synaptonemal complex segment was not identified. From mid pachytene onwards the $\mathrm{XY}$ bivalent is frequently associated with material resembling a 


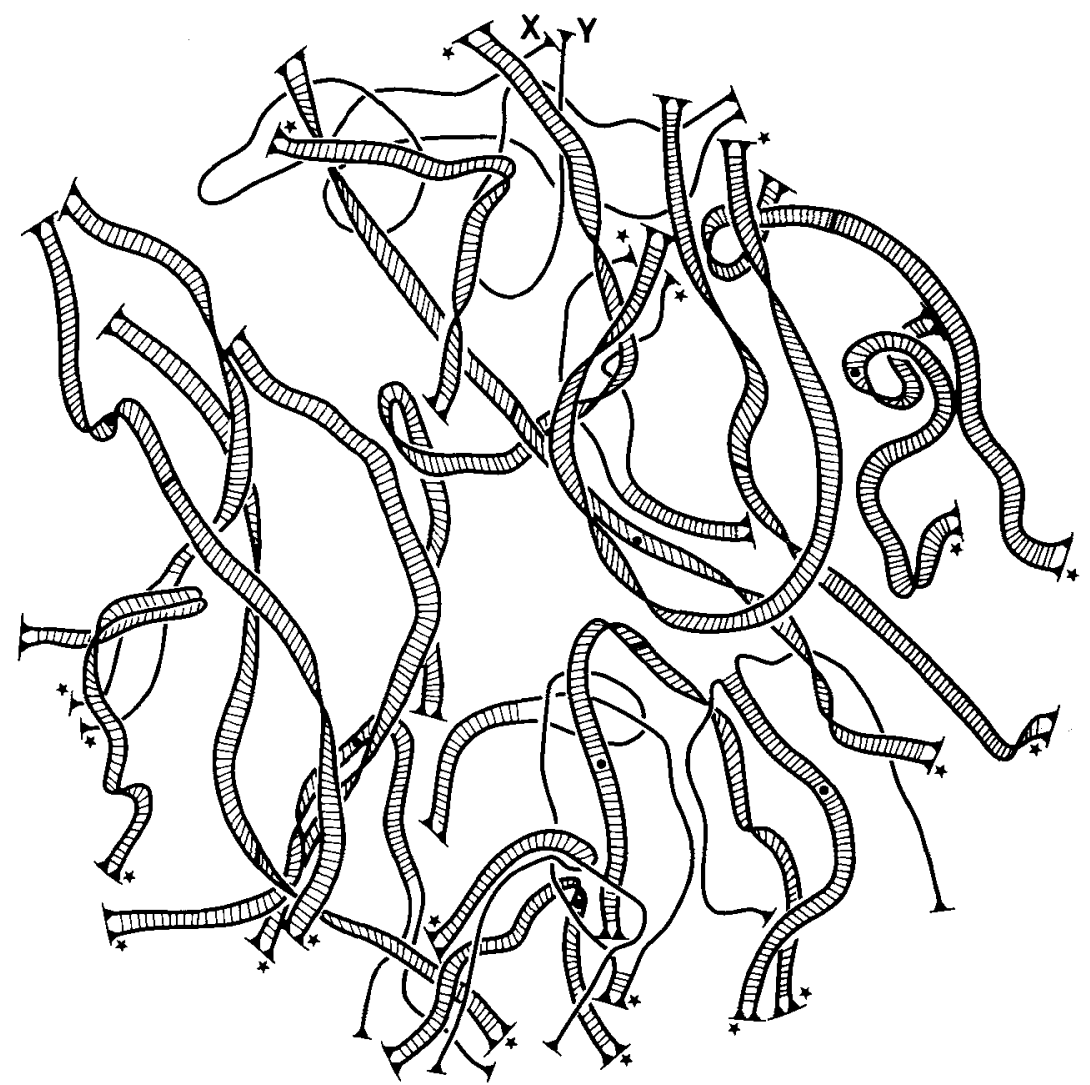

Figure 8. A reconstruction of an early diplotene nucleus. An explanation to the symbols is given in the legend to Figure 2.

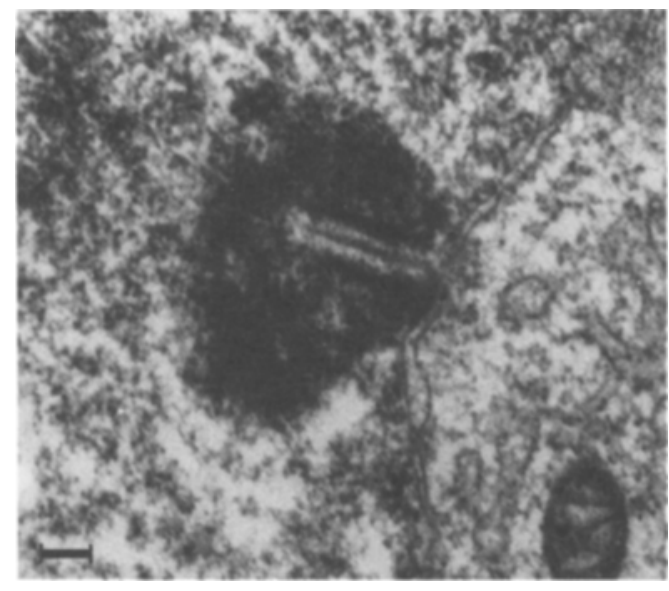

Figure 9. A micrograph showing the centromeric heterochromatin. (Bar $=0,1 \mu \mathrm{m})$.

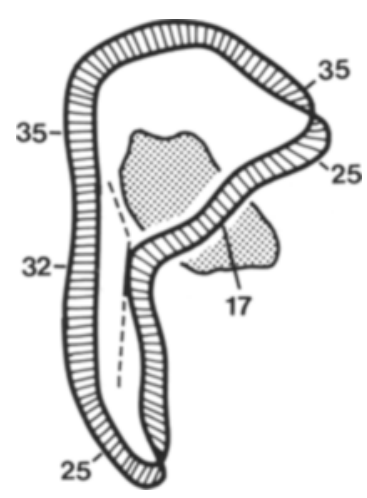

Figure 10. A reconstruction of the ring bivalent observed in a late pachytene nucleus. The dark hatching denotes the position of the centromere. The nuclear envelope is marked by the dashed line. The figures denote the section numbers of the nucleus. 


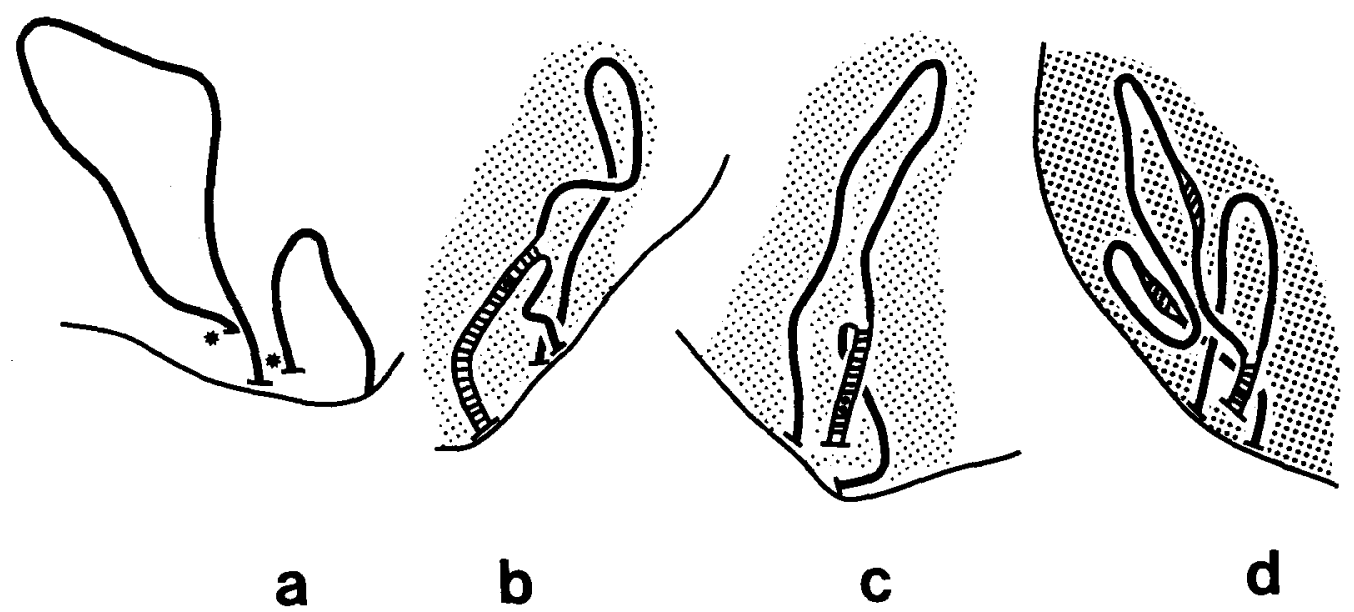

Figure 11. Reconstructions of the $X$ and $Y$ chromosomes at late zygotene (a), early pachytene (b), mid pachytene (c), and late pachytene (d). An explantation to the symbols is given in the legend to Figure 2.

nucleolus, as previously reported for the mouse $(36,37)$ and for human pachytene spermatocytes $\left(\begin{array}{lll}17 & 18,30\end{array}\right)$.

\subsubsection{The nucleoli}

Two to five nucleoli are present in each pachytene nucleus. Two distinctly different nucleolus conformations are seen, one type consisting largely of thick threads of nucleolar material, nucleonema (Figure 5) while the other type is spherical and compact (Figure 7). The former type is probably associated with actively transcribing ribosomal cistrons, while the latter type intimates an inactive nucleolus organizer region. As in human spermatocytes, the nucleoli are associated with the centromeric heterochromatin through a spherical fibrillar body (17).

In human spermatocytes all five nucleolar organizing chromosome pairs are associated with a nucleolus at late zygotene-early pachytene. Thereafter, the number of nucleoli decreases and their structure changes from the open and apparently active reticulate form to a spherical, compact, and probably inactive form. A similar development is not apparent in the present study, and generally it is not possible to identify all the nucleolus organizing chromo- somesdue to extensive aggregation of the centromeric heterochromatin of several bivalents. Hence, neither the number of active nucleolus organizing chromosomes, nor the number and structure of nucleoli can be used as reliable markers of the developmental stage of the nuclei.

\subsection{Recombination nodules and bars}

\subsubsection{The structure and number of nodules and bars}

Three different recombination structures have been detected in the present study: 1) Recombination nodules, i.e., dense spheres associated with the central region of the synaptonemal complex (Figure 12a). 2) Recombination bars, i.e., fusiform, dense structures bridging the central region, and lying at a right or oblique angle to the lateral components (Figure 12d). 3) T-shaped structures which appear to be intermediates between nodules and bars (Figure $12 \mathrm{~b}$, c). In the quantitative analysis the $\mathrm{T}$-shaped structures are counted as nodules.

The mean number of nodules and bars per nucleus for each of the three substages of pachytene is presented in Table I together with the number observed in the late zygotene and the early diplotene nuclei. The data reveal a decrease 
Table II. The length of the synaptonemal complex segment (SC) between the $\mathrm{X}$ and $\mathrm{Y}$ chromosomes at pachytene and the number and position of recombination nodules (RN) or recombination bars (RB) in the paired segment. sd, standard deviation.

\begin{tabular}{|c|c|c|c|c|c|c|}
\hline \multirow[t]{2}{*}{ STAGE } & \multirow[t]{2}{*}{$\begin{array}{l}\text { Nucleus } \\
\text { number }\end{array}$} & \multirow[t]{2}{*}{$\begin{array}{l}\text { SC length } \\
(\mu \mathrm{m})\end{array}$} & \multirow{2}{*}{$\begin{array}{l}\text { type of } \\
\text { recombin. } \\
\text { structure }\end{array}$} & \multirow{2}{*}{$\begin{array}{l}\text { distance between } \\
\text { RN/RB and telomer } \\
(\mu \mathrm{m})\end{array}$} & \multicolumn{2}{|c|}{$\begin{array}{l}\text { length of } X \& Y \\
\text { lateral components }\end{array}$} \\
\hline & & & & & $X$ & $Y$ \\
\hline Early & 17 & 0 & - & - & 9.3 & 5.6 \\
\hline \multirow[t]{10}{*}{ pachytene } & 18 & 0.9 & RN & 0.4 & 7.6 & 2.8 \\
\hline & 19 & 1.5 & $\mathbf{R N}+\mathbf{R N}$ & 0.4 & 9.3 & 3.2 \\
\hline & 20 & 0.8 & none & - & 8.4 & 3.9 \\
\hline & 21 & 2.3 & RN & 1.8 & 9.7 & 3.9 \\
\hline & 22 & 0.5 & none & - & 8.1 & 3.8 \\
\hline & 23 & 1.4 & RN & 0.4 & 8.1 & 3.6 \\
\hline & 24 & 0.7 & RN & 0.6 & 10.2 & 3.5 \\
\hline & 25 & 1.6 & RN & 0.2 & 7.9 & 3.4 \\
\hline & 30 & 0.7 & RN & 0.6 & 7.1 & 3.1 \\
\hline & 31 & 0 & - & - & 8.0 & 3.0 \\
\hline Mean \pm sd & & $1.2 \pm 0.9$ & & $0.6 \pm 0.5$ & & \\
\hline & 7 & 0.9 & $\mathrm{RN}$ & 0.5 & - & - \\
\hline \multirow{5}{*}{ pachytene } & 8 & 0 & - & - & 6.5 & 2.8 \\
\hline & 10 & 0.7 & RN & 0.4 & 8.2 & 3.8 \\
\hline & 11 & 0.5 & none & - & 6.4 & 4.0 \\
\hline & 12 & 0.3 & none & - & 6.7 & 3.0 \\
\hline & 13 & 0.8 & RN & 0.5 & 8.7 & 5.5 \\
\hline Mean \pm sd & & $0.6 \pm 0.2$ & & $0.4 \pm 0.1$ & & \\
\hline & 1 & 0.2 & RN & 0.2 & 11.3 & 5.0 \\
\hline \multirow{6}{*}{ pachytene } & 2 & 0.2 & RN & 0.1 & 10.3 & 5.0 \\
\hline & 3 & 0.2 & none & - & 12.5 & 5.6 \\
\hline & 4 & 0.5 & $\mathrm{RB}$ & 0.2 & 14.9 & 5.3 \\
\hline & 5 & 0.2 & none & - & 11.9 & 5.9 \\
\hline & 6 & 0.5 & RN & 0.1 & 14.2 & 5.2 \\
\hline & 26 & 0 & - & - & 9.8 & 4.0 \\
\hline Mean \pm sd & & $0.3 \pm 0.1$ & & $0.2 \pm 0.0$ & & \\
\hline
\end{tabular}
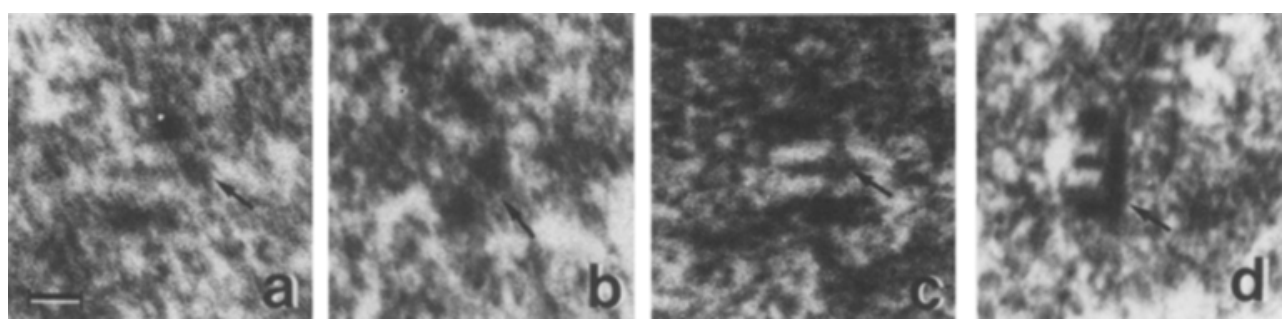

Figure 12. A series of micrographs showing spherical (Figure 10a) and "T"-shaped (Figure 10b, c) recombination nodules and a recombination bar (Figure $10 \mathrm{~d})$. (Bar $=0,05 \mu \mathrm{m})$. 
TABLE III. Distribution of recombinational structures among the bivalents. Observed frequencies are compared to the expected frequencies if the nodules/bars are distributed randomly among the bivalents.

\begin{tabular}{|c|c|c|c|c|c|c|}
\hline \multirow[b]{2}{*}{ STAGE } & \multicolumn{6}{|c|}{ Percent bivalents with $\mathrm{n}$ nodules/bars } \\
\hline & $\mathrm{n}=0$ & 1 & 2 & 3 & 4 & 5 \\
\hline \multicolumn{7}{|c|}{ Early pachytene } \\
\hline observed & 13 & 35 & 30 & 16 & 5 & 1 \\
\hline expected & 19 & 31 & 26 & 14 & 7 & 2 \\
\hline \multicolumn{7}{|c|}{ Mid pachytene } \\
\hline observed & 43 & 41 & 16 & 0 & 0 & 0 \\
\hline expected & 48 & 35 & 13 & 3 & 0.5 & \\
\hline \multicolumn{7}{|c|}{ Late pachytene } \\
\hline observed & 44 & 42 & 11 & 3 & 0 & 0 \\
\hline expected & 50 & 33 & 12 & 4 & 1 & \\
\hline
\end{tabular}

of the total number of recombination structures from 33 per nucleus at early pachytene to 14 at mid and late pachytene. This numerical change is accompanied by a change in the nodule/bar ratio. A few bars are present at early pachytene while at mid and late pachytene the nodules and bars are about equally frequent. This is also the case in the reconstructed early diplotene nucleus.

\subsubsection{The distribution of recombination structures among the bivalents}

The observed distributions of recombination structures (nodules and bars) among the bivalents at early, mid, and late pachytene are compared with the distributions expected if the nodules and bars are placed at random among the bivalents. The random distributions have been constructed by computer simulation as described in detail in (30) and summarized below: The absolute lengths of the individual bivalents of all the nuclei are fed into a computer which has been programmed to position, one at a time the number of nodules observed in each nucleus randomly along and among the bivalents. This procedure is repeated a number of times for each nucleus, the total number of runs for each substage being approximately 10,000.

A comparison between the observed distributions of recombination structures among the bivalents and the distributions obtained by placing nodules and bars at random is presented in
Table III. At all three substages more bivalents than expected possess one or two recombination nodules whereas the frequency of bivalents devoid of nodules and bars is lower than expected if recombination structures were randomly distributed.

\subsubsection{Neighbour nodule/bar and telomere- nodule/bar distances}

An analysis of the distribution of recombination structures relative to each other and relative to the telomeres is carried out by comparing the frequencies of observed distances (in $0.3 \mu \mathrm{m}$ intervals) with frequencies obtained from the computer simulation experiment where nodules/bars are placed at random. The following conclusions may be drawn from this comparison (Figure 13): 1) At early pachytene the recombination structures are distributed independently of each other as judged by the virtual identity between the observed distribution and the distribution obtained by random placement of nodules and bars. At mid and late pachytene this is no longer the case, there being a deficit of short neighbour nodule/bar distances and an excess of longer distances in particular from $1.5-2.0 \mu \mathrm{m}$ at mid and late pachytene, from 3.0-3.6 $\mu \mathrm{m}$ and from 4.5-5.2 $\mu \mathrm{m}$ at mid pachytene, and distances longer than $5.7 \mu \mathrm{m}$ at late pachytene. 2) The distribution of the nodules/ bars relative to the telomers basically follows the pattern described for neighbour-nodule distances. 


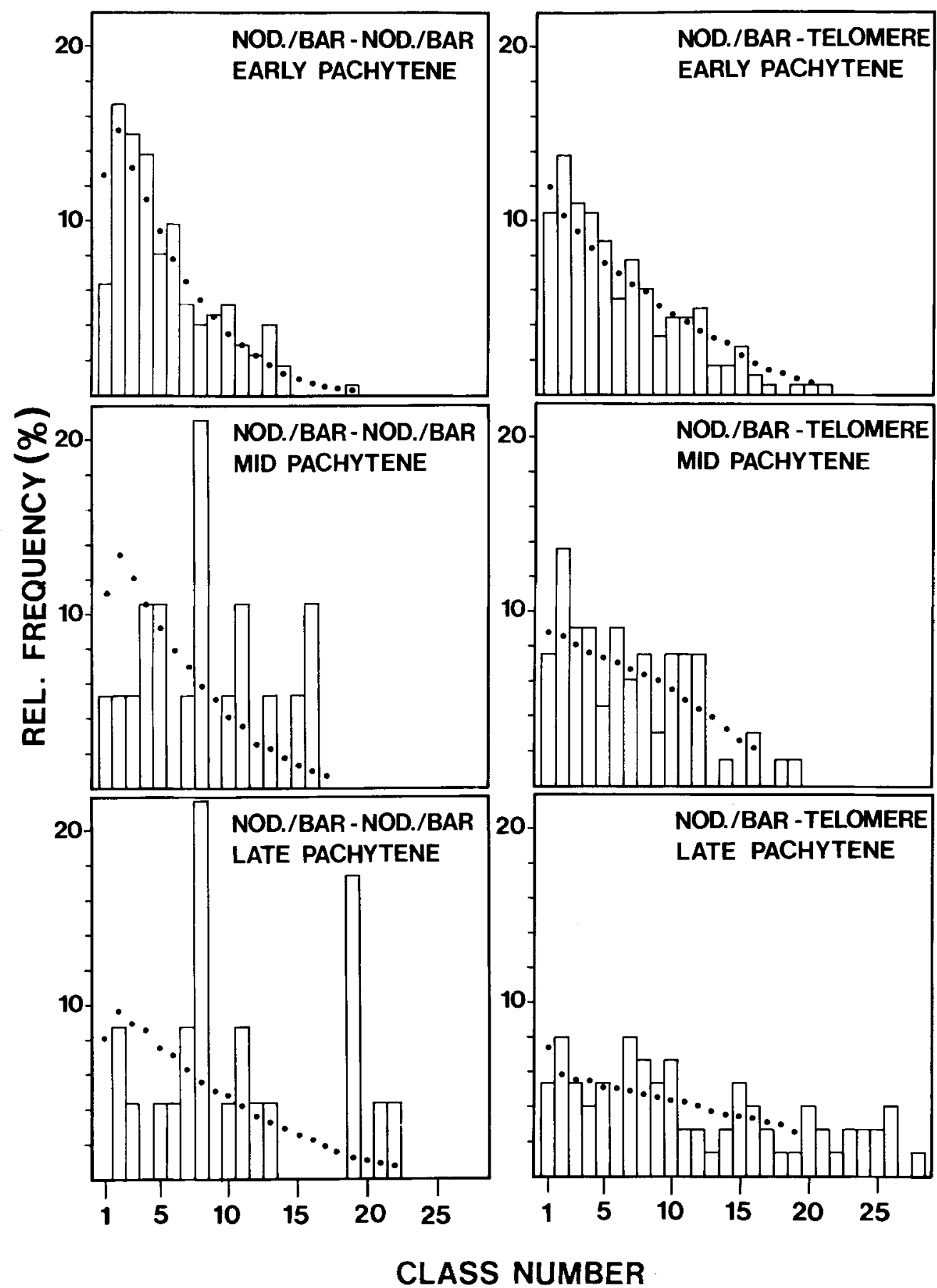

Figure 13. The distribution of the distances between the telomere and the first nodule or bar (right column) and the distance distribution for neighbour nodule/bar (left column) at early, mid and late pachytene. The histograms show the distribution of the observed distances expressed as relative frequencies of distances in $0.3 \mu \mathrm{m}$ classes. The dots represent the relative frequencies expected if nodules and bars were distributed at random among and along the bivalents. 

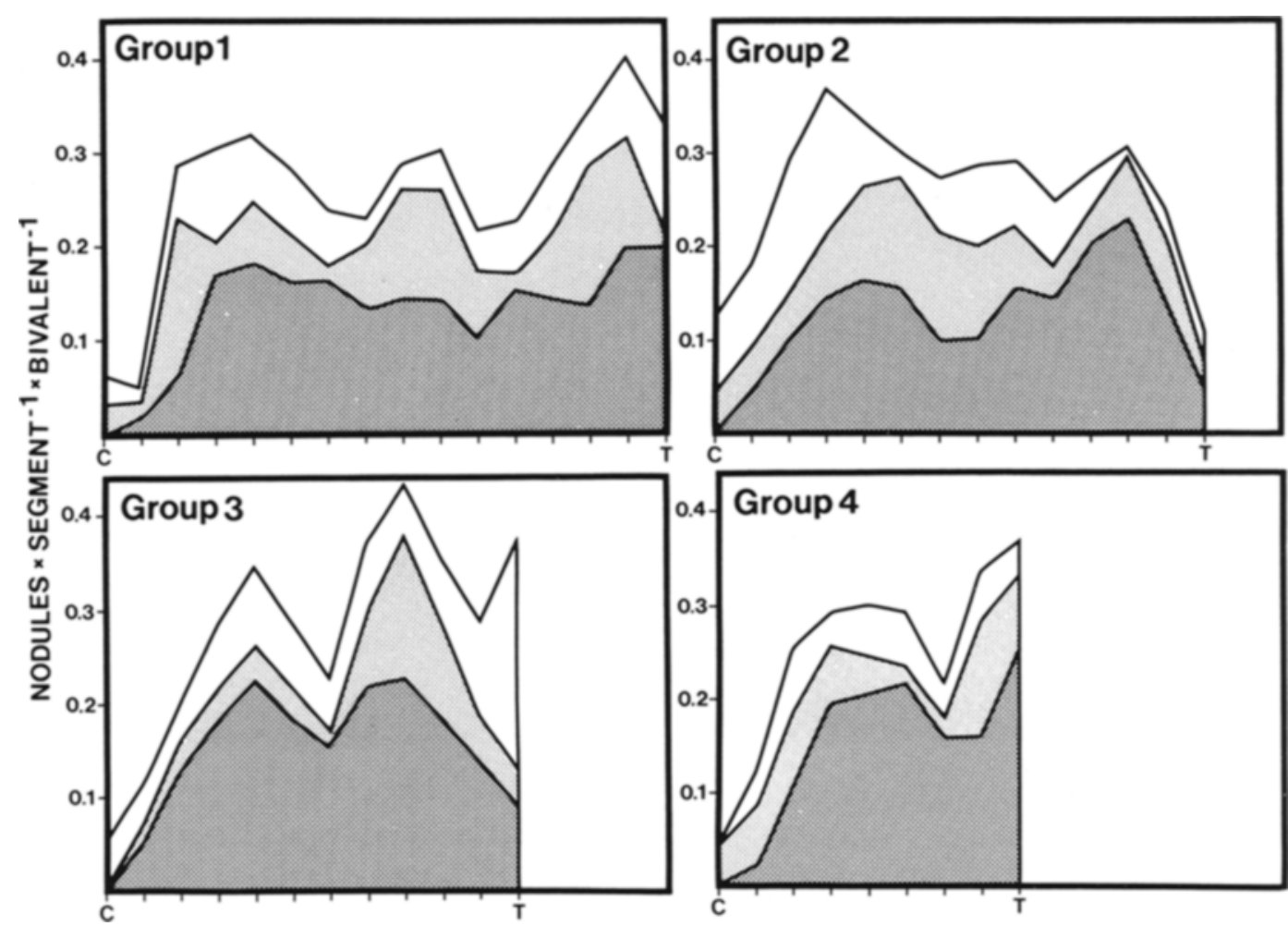

Figure 14. The cumulative distribution of recombinational structures along the bivalents at early pachytene (dark hatching), early + mid pachytene (light hatching) and early + mid + late pachytene (no hatching). The frequencies plotted in the diagram are the mean of two adjacent intervals (each equal to $0.5 \mu \mathrm{m}$ ) except for the telomeric and centromeric segments which are presented by their actual value.

\subsubsection{Distribution of nodules and bars along the bivalents}

Since all the bivalents are acrocentric and none of them carry morphological marker structures, it is not possible in the present study to establish the specific identity of any of the 19 autosomal bivalents, beyond a simple ranking according to synaptonemal complex length. Such a ranking reveals a continuous variation in size and a particular chromosome may thus receive a different number in different nuclei. In order to determine the distribution of nodules and bars along the bivalents, the bivalents of each nucleus are ranked and numbered after decreasing length and divided into four groups: group 1, the four longest bivalents; group 2, bivalents numbers 5-9; group 3, bivalents num- bers 10-15; group 4, bivalents numbers 16-19.

The lengths of the bivalents in each group are adjusted to the mean pachytene length for the group, maintaining the relative positions of nodules and bars. The number of nodules and bars of each substage is then counted in $0.5 \mu \mathrm{m}$ intervals along the bivalents in each group. To facilitate the interpretation, the frequencies, (i.e., the number of nodules/bars per interval per nucleus) plotted in the diagram are the mean of two adjacent intervals except for the values at the telomeres which are represented by their actual value.

The data in Figure 14 are presented as cumulative frequencies obtained by adding the frequencies in each interval to those found at previous stages. The following conclusions may 


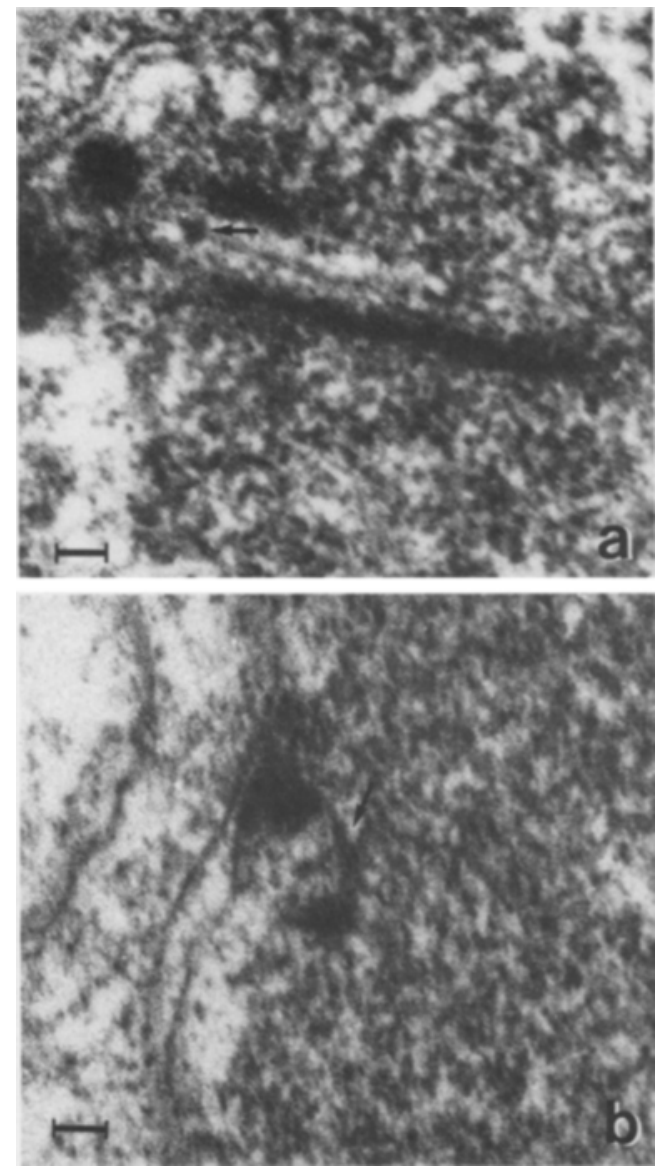

Figure 15. Micrographs of XY bivalents. The recombination nodule in Figure 15a and the recombination bar in Figure $15 \mathrm{~b}$ are denoted by arrows. (Bar $=0,05 \mu \mathrm{m}$ ).

be drawn from this analysis: 1) The distribution of nodules and bars is the same at all three substages of pachytene. 2) Each group of bivalents exhibits a characteristic pattern of peaks and troughs which relates to the length of the bivalents. The longer bivalents (group 1) possess three peaks while the shorter bivalents (groups 3 and 4) contain two peaks. In group 2 three less prominent peaks are apparent. 3) Nodules and bars are rare in segments flanking the centromeres while their highest frequency is attained subterminally in the non-centromeric end of the bivalents.

\subsubsection{Nodules and bars associated with the $X Y$ bivalent}

At early pachytene recombination nodules or bars (Figure 15) are present in seven of the nine $X Y$ bivalents containing a synaptonemal complex segment (Table II). With only one exception (nucleus number 21 ) the recombination structures are confined to the distal $0.6 \mu \mathrm{m}$ of the synaptonemal complex. At mid and late pachytene recombination structures in the form of bars and T-shaped intermediates are seen in 60\% and $67 \%$ of the segments, respectively. At mid and late pachytene the synaptonemal complex length in the $X Y$ bivalent decreases and the location of the nodule or bar is more distal. At late pachytene the nodule/bar is found in the distal $0.2 \mu \mathrm{m}$ of the segment. In the early diplotene nucleus a synaptonemal complex can not be seen. The telomeres of the noncentromeric ends of the $\mathrm{X}$ and $\mathrm{Y}$ chromosomes are, however, aligned at a distance corresponding to the width of a central region.

\section{DISCUSSION}

\subsection{Substages of pachytene}

Complete reconstructions of one late zygotene nucleus, 24 pachytene nuclei and one diplotene nucleus has permitted a subdivision of the pachytene stage in mouse spermatocytes into an early, a mid, and a late substage. The observations presented in section 3.1 have revealed a series of continuous changes in nuclear fine structure from late zygotene to early diplotene, and it is reasonable to conclude that the developmental sequence covers the entire pachytene period.

In a previous study of human spermatocytes (17), ultrastructural parameters such as stages of centriole duplication, ultrastructure of centromeres, and number and morphology of nucleoli proved useful in a subdivision of the pachytene stage in addition to the characteristics used in the present study (i.e., bouquet formation, chromatin organisation and density, and the XY bivalent). However, neither centriole organisation nor the centromeric heterochromatin appear to change during the pachytene stage in the mouse spermatocytes and as described in section 3.2.4, nucleoli with the same fine structure are observ- 
ed throughout pachytene. It turns out to be impossible to determine the exact number of nucleolus organizing chromosomes due to the aggregation of the centromeric heterochromatin of different bivalents. The observed maximum of five nucleoli indicates that at least five nucleolar organizing bivalents are present. Using a silver staining procedure OUD and REUTLINGER (25) identify nucleolus organizing regions on chromosomes $12,15,16,17$ and 18 . In situ hybridization demonstrates ribosomal DNA cistrons on chromosomes 15,18 and 19(9) and on chromosomes 12, 16 and 18 (14). In quinacrine mustard-stained mitotic metaphase nuclei the number of secondary constrictions appears to be strain specific, the maximum of easily identifiable constrictions being five per genome (5).

The developmental sequence of the pachytene nuclei reported above agrees well with that found in other studies of mouse spermatocytes. The ultrastructural changes in chromatin condensation and in the morphology of the $X Y$ bivalent during pachytene are very similar to those observed by SolaRI $(36,37)$ from sectioned material or from spread nuclei $(6,7,8,12$, 26, 32). POORMAN et al. (28) distinguish five substages of pachytene partly based on the extent of synapsis of the $\mathrm{X}$ and $\mathrm{Y}$ chromosomes and the morphology of the $X Y$ body. The duration of the stages is estimated from spreads and by pulse-chase experiments with ${ }^{3} \mathrm{H}$-thymidine (23). The total duration of pahytene is approximately 162 hours, in agreement with previous estimates suggesting the duration of the pachytene stage to be 7-8 days (11).

The present study has shown that the length of the synaptonemal complex increases from 104 $\mu \mathrm{m}$ at mid pachytene to $159 \mu \mathrm{m}$ at late pachytene. Based on surface spread nuclei Moses (23) finds in both oocytes and spermatocytes a pronounced decrease $(50 \%)$ in synaptonemal complex length between zygotene and diplotene, the length at late pachytene in the male amounting to $162 \mu \mathrm{m}$. This discrepancy most likely is the result of the two different methods of preparation and indicates considerable stage specific stretching of the complement caused by surface spreading.

\subsection{The morphological and numerical changes of recombination structures}

Both recombination nodules and bars are present in mouse spermatocytes. At late zygotene only nodules are found while the first bars appear at early pachytene. At mid and late pachytene the two types are equally frequent. Since recombination structures with an intermediate $\mathrm{T}$-shape are frequent it seems likely that recombination bars evolve from recombination nodules.

These results are identical to those obtained in human spermatocytes where the first bars appear during the second half of early pachytene. A morphological change from nodules to bars has also been reported for human oocytes, although here the bars appear already during late $z y$ gotene. Recombination bars (lateral element cross connections - $\mathrm{CO}$ elements) are likewise present at pachytene stage VII in rat spermatocytes (22). Structural changes of recombination nodules from smaller and less dense structures at zygotene to larger and denser structures at mid pachytene have furthermore been described in Bombyx, Coprinus and Drosophila $(16,19,4)$. In the latter organism intermediates between nodules and larger structures are extremely rare and the possibility that the small and large nodules constitute independent pools has been considered.

In the human male, the morphological differentiation of recombination nodules into bars coincides with a reduction in number and a change from a random distribution into a distribution similar to that of chiasmata. This evidence has led to the conclusion (18) that the morphological change signifies the occurrence of crossing over. Because of the great similarity in nodule behavior and morphology between mouse and man, it is conceivable that the occurrence of crossing over in the mouse is also signalled by the conversion of the nodule into a bar.

In the mouse the number of nodules/bars decreases from a mean of 33 at early pachytene to 14 at mid and late pachytene. A similar reduction occurs in human spermatocytes (18), but in contrast to the situation in human spermatocytes, where all nodules and bars have disappeared by early diplotene, the frequency of 
recombination structures in mouse spermatocytes is the same at mid and late pachytene and possibly also in early diplotene. A substantial number of nodules and bars are also present in human oocytes at the early diplotene stage (3).

The presence of a substantial number of nodules and bars at diplotene indicates that crossing over may still occur at this stage, despite the fact that synaptonemal complex elimination has commenced in other segments of the bivalents.

\subsection{The distribution of recombination} structures among and along the bivalents

The analysis of the distribution of recombination nodules and bars among the bivalents has shown that at all three substages of pachytene there is an excess of bivalents with one and two nodules/bars whereas bivalents without nodules are less frequent than expected if the nodules/ bars were distributed at random. Hence, in mouse as in all other organisms investigated so far, mechanisms exist which tend to ensure each bivalent at least one nodule. However, in most of these organisms (e.g., human spermatocyte, Bombyx spermatocytes, Coprinus meiocytes, Drosophila oocytes) a more drastic change is apparent when the distribution at late zygotene and early pachytene is compared with that at mid pachytene. The distribution at early pachytene is nearly random while there is a marked deviation from randomness at mid pachytene where virtually all bivalents have at least one nodule/bar. Less pronounced distributional changes are found in the human oocytes (3). One might speculate that crossing over in mouse spermatofytes and human oocytes is a less synchronous process than for example in human spermatocytes where the majority of crossing overs appears to take place at mid pachytene. This may in mouse spermatocytes and human oocytes reduce the distributional differences between successive stages.

The reduction in the number of recombination structures from early to mid pachytene is accompanied by a change in the distribution of neighbour nodule distances. At early pachytene nodules/bars are located virtually at random while two or more nodules are associated with the synaptonemal complex at preferred distances during mid pachytene, short distances being rare. This implies that originally closely positioned nodules/bars are more susceptible to elimination than nodules/bars located farther away from each other. The placement of nodules/bars at particular distances is also reflected in the distribution of nodules along the bivalents. The shape of the cumulative frequency distribution reveals a clear pattern of peaks and troughs, the number of peaks being related to the length of the bivalent. Since the data are pooled for similar sized bivalents, the interpretation of the distribution rests on the assumption that the distribution of nodules and bars along the bivalent primarily is a function of its length and not a unique property of the individual bivalent. This assumption seems valid in human spermatocytes and oocytes where the distribution of nodules and bars can be characterized for individual bivalents. Although some variation is encountered between similar sized bivalent arms, a basic pattern relating to the length of the arms could be identified $(3,18)$. This is also apparent in the present analysis where the number of peaks and troughs correlates well with the mean bivalent length of each group. In addition, the frequency of nodules/bars is low near the centromere region and high in regions close to the telomeres, a phenomenon also seen in human oocytes (2) and spermatocytes (18).

\subsection{The total number of crossovers}

Since only some of the recombination nodules mediating crossing over are present at a given stage the total number of crossovers cannot be counted directly. A minimum estimate of the total number of crossover events can, however, be made using the mean cumulative nodule/bar frequency of the shortest chromosome arms (group 4) as a standard of one chiasma. The total number of crossovers per nucleus can then be determined by dividing the cumulative nodule frenquency for all bivalents by the frequency corresponding to one chiasma. Estimated in this way, the total number of crossovers per nucleus amounts to 35 . This estimate rests on the assumption that the time required for a nodule to transform into a bar and subsequently to disap- 
pear is constant, and that the fraction of recombination nodules which actually leads to crossing over is the same in all bivalents at all stages.

Thirty-five crossovers per nucleus is somewhat higher than that found in genetic studies (map length: $1250 \mathrm{cM}$ : corresponding to 25 crossovers/nucleus) (13) and by chiasma counts in the light microscope (22 to 26 chiasmata per nucleus) $(20,27,33,34)$. However, the genetic map length is probably underestimated due to lack of terminal markers. Furthermore, terminalization may cause a reduction of the number of recognizable chiasmata. A similar discrepancy is observed between crossing over frequencies predicted from the cumulative nodule distributions and the number of chiasmata at diakinesis in human oocytes and spermatocytes, whereas there is good correspondance between the estimated number of crossovers and the number of retained synaptonemal complex segments, i.e., the early chiasmata, at diplotene (18). Unfortunately, quantitative data on the number of diplotene chiasmata are not at hand in mouse spermatocytes, and a more precise characterization of the total number of crossovers in the mouse genome therefore awaits further investigations.

\subsection{Chromosome pairing and crossing over between the $\mathrm{X}$ and $\mathrm{Y}$ chromosomes}

The $\mathrm{X}$ and $\mathrm{Y}$ chromosomes appear to pair late compared to the autosomes. At early pachytene synaptonemal complex formation between the $\mathrm{X}$ and $\mathrm{Y}$ chromosomes reaches its maximum, up to $55 \%$ of the long arm of the $Y$ chromosome being paired with the $\mathrm{X}$ chromosome. Nearly all $X Y$ bivalents possess one or rarely two recombination nodules which with a single exception were located within the distal $0.6 \mu \mathrm{m}$ segment. At mid and at late pachytene, the synaptonemal complex is considerably shorter or in a few cases absent. A recombination structure is present in two-thirds of the segments. At early diplotene the synaptonemal complex is completely dismantled.

A late pairing and synaptonemal complex formation between the sex chromosomes appear to be the rule in mammalian spermatocytes possibly due to the combined effect of the condensation of the $X$ and $Y$ chromosomes into the $X Y$ body and, as discussed below, the fact that homology between the sex chromosomes appears only to involve the most distal parts of the long arms. Hence fewer recognition sites are available for the alignment of the lateral elements. Subsequent extention of the synaptonemal complex during early pachytene will thus occur between non-homologous segments.

A progressive desynapsis of the $\mathrm{X}$ and $\mathrm{Y}$ chromosome reported in a number of investigations on mouse spermatocytes is confirmed in the present study. The variation in length of the synaptonemal complex segments is, however, substantial between nuclei at the same substage as defined by the criteria listed in section 3.1 , and the length of the paired segment can not by itself, but only in combination with other parameters, be used as a reliable marker of a pachytene substage.

Recent observations in the mouse have provided strong support for the classical contention of an obligate crossover between the $\mathrm{X}$ and $\mathrm{Y}$ chromosomes. Evans et al. (10) demonstrated that male determining genes, translocated to the tip of the long arm of the $Y$ chromosome, were transferred to the $\mathrm{X}$ chromosome by means of an obligate crossover resulting in sex reversal in the progeny. KEITGES et al. (21) have likewise demonstrated that a mouse steroid sulphatase variant appears to be present distal to the site for an obligate crossover between the sex chromosomes. In contrast, all other genetic and structural markers on the $\mathrm{X}$ chromosome including distal markers in the long arm are $\mathrm{X}$-linked showing that the obligate crossover is close to the telomere of the $\mathrm{X}$ and $\mathrm{Y}$ chromosomes (10).

The presence of recombination nodules and bars at a distal location in the paired segment of the $\mathrm{X}$ and $\mathrm{Y}$ chromosomes is in excellent agreement with these results. If taken at face value, the nodule/bar frequencies at mid and late pachytene indicate that $30-40$ percent of the $X Y$ bivalents do not undergo crossovers. It is considered likely, however, that all crossovers in the $\mathrm{XY}$ bivalent cannot be recognized as bars at the same time as is the case for the autosomes, and that all XY bivalents form a crossover with the aid of a nodule. 
The present data are in good correspondance with those reported for the $\mathrm{XY}$ bivalent in man $(18,39)$. Reconstruction of 33 early pachytene nuclei revealed one or two recombination nodules in $75 \%$ of the XY bivalents. Towards the end of early pachytene intermediate $\mathrm{T}$-shaped nodules were observed indicating the transformation of the nodule into a bar.

Seventy-five percent of the nodules were located distally in the synaptonemal complex segment combining the sex chromosomes at a distance corresponding to one third of the short arm of the Y-chromosome. During diplotene a short synaptonemal complex chiasma was retained between the short arms of the $\mathrm{X}$ and $\mathrm{Y}$ chromosomes.

In agreement with these observations, molecular studies by ROYER et al. (31) have recently identified homologous sequences distally in the short arms of the sex chromosomes. The most distal genes recombined with a frequency of $50 \%$ indicating the occurrence of an obligatory crossover between the sex chromosomes.

\section{ACKNOWLEDGEMENTS}

I am highly indebted to Professor DiTER VON WETTSTEIN for suggesting this project and to let me work in the department. I owe a special debt to Dr. P.B. HoLM and Dr. S.W. RASMUSSEN for excellent supervision and for never failing interest in my work. JEAN SAGE is kindly thanked for technical assistence and NINA RASMUSSEN for drawing the diagrammes. Financial support was provided by grant BIO-E-417 DK (G) from the Commision of European Communities.

\section{REFERENCES}

1. BAKer. B.S., A. T. C. CARPENTER, M.S. Esposito, R.E. ESPOSITO\& L. SANDER: The genetic control of meiosis. Ann. Rev. Genet. 10, 53-134 (1976)

2. BoJKo, M: Human meiosis VIII: Chromosome pairing and formation of the synaptonemal complex in oocytes. Carlsberg Res. Commun. 48, 457-483 (1983)

3. Bosko, M.: Human meiosis IX. Crossing over and chiasma formation in oocytes. Carlsberg Res. Commun. 50, 43-72 (1985)

4. CARPenter, A. T. C.: Recombination nodules and synaptonemal complex in recombination defec- tive females of Drosophila melanogaster. Chromosoma 75, 259-292 (1979)

5. Dev. V. G., M. S. Grewal, D. A. Miller, R. E. KOURI, J. J. HutTon \& O. J MiLler: The quinacrine flourescence karyotype of Mus Musculus and demonstration of strain differences in secondary constrictions. Genetica 10,436-451 (1971)

6. Dietrich. A. J. J. \& P. DE BOER: A sequential analysis of the development of the synaptonemal complex in spermatocytes of the mouse by electron microscopy using hydroxyurea and agar filtration. Genetica 61, 119-129 (1983)

7. Dietrich. A. J. J. \& R. J. P. Mulder: A light microscopic study of the development and behaviour of the synaptenemal complex in spermatocytes of the mouse. Chromosoma 83, 409-418 (1981)

8. DResSER. J. W. \& M. J. MOSES: Silver staining of synaptonemal complexes in surface spreads for light microscopy and electron microscopy. Exp. Cell. Res. 121, 416-419 (1979)

9. ELSEVIER. S. M.\& F. H. RUDDLE: Location of genes coding for the $18 \mathrm{~S}$ and $28 \mathrm{~S}$ ribosomal RNA within the genome of Mus musculus. Chromosoma 52, 219-228 (1975)

10. Evans, E. P. M. D. Burtenshaw \& B. M. CatTANACH: Meiotic crossing-over between the $X$ and $Y$ chromosomes of male carrying the sexreversing (Sxr) factor. Nature 300, 443-445 (1982)

11. Ghosal, S. K. \& B. B. Mukherjee: The chronology of DNA synthesis and spermatogenesis in the male mouse and golden hamster. Can. J. Genet. Cytol. 13, 672-682 (1971)

12. Guitart, M., M. D. Coll, M. Ponsa \& J. EgozCUE: Sequential study of synaptonemal complexes in mouse spermatocytes by light and electron microscopy. Genetica 67, 21-30 (1985)

13. Green, M. C.: The laboratory mouse Mus musculus. In: Handbook of Genetics, e. d. R. C. King 4, 203-241, Plenum Press, New York, London (1975)

14. Henderson, A. S., E. M. EICHER, M. T. Yu \& K. C. ATwOOD: The chromosomal location of ribosomal DNA in the mouse. Chromosoma 49, 155 160 (1974)

15. Holm.P. B. \& S. W. Rasmussen: Human meiosis I. The human pachytene karyotype analyzed by three dimensional reconstruction of the synaptonemal complex. Carlsberg Res. Commun. 42, 283-323 (1977)

16. Holm. P. B. \& S. W. Rasmussen: Chromosome pairing, recombination nodules and chiasma formation in diploid Bombyx males. Carlsberg Res. Commun. 45, 483-458 (1980)

17. Holm, P. B. \& S. W. Rasmussen: Human meiosis 
V. Substages of pachytene in human spermatogenesis. Carlsberg Res. Commun. 48, 351-383(1983)

18. Holm, P. B \& S. W. Rasmussen: Human meiosis VI. Crossing over in human spermatocytes. Carisberg Res. Commun. 48, 385-413 (1983)

19. Holm, P. B., S. W. Rasmussen, D. Zickler, B. C. LU \& J. SAGE: Chromosome pairing, recombination nodules and chiasma formation in the basidiomycete Coprinus cinereus. Carlsberg Res. Commun. 46, 305-346 (1981)

20. JaGiello, G. \& J. S. FANG: Analysis of diplotene chiasma frequencies in mouse oocytes and spermatocytes in relation to ageing and sexual dimorphism. Cytogenet. Cell Genet. 23, 53-60 (1979)

21. Keitges, E., M. Rivest. M. Siniscalco \& S. M. GARTLER: X-linkage of steroid sulphatase in the mouse is evidence for a functional $Y$-linked allele. Nature 315, 226-227 (1985)

22. MOENS, P. B.: Lateral element cross connections of the synaptonemal complex and their relationship to chiasmata in rat spermatocytes. Can. J. Genet. Cytol. 20, 567-579 (1978)

23. Moses, M. J.\& P. A. Poorman: Synapsis, synaptic adjustment and DNA synthesis in mouse oocytes. In: Chromosomes Today, ed. M. D. Bennett, A. Gropp and U. Wolf. Volume 8, 90-103 (1984)

24. Moses, M. J., P. A. Poorman, T. H. Roderick \& M. T. DAviSSON: Synaptonemal complex analysis of mouse chromosomal rearrangemnts IV. Synapsis and synaptic adjustment in two paracentric inversions. Chromosoma 84, 457-474 (1982)

25. OUD, J. L. \& A. H. H. ReuTlinger: The behaviour of silver-positive structures during meiotic prophase of male mice. Chromosoma $81,569-578$ (1981)

26. Pathak, S.\&T.C.Hsu:Silver-stained structures in mammalian meiotic prophase. Chromosoma 70 , 195-203 (1979)

27. POlani, P. E.: Centromer localization at meiosis and the position of chiasmata in the male and female mouse. Chromosoma 36, 343-374 (1972)

28. Poorman, P.A. M.J. Moses. M.T. Davisson \& T. H. RODERICK: Synaptonemal complex analysis of mouse chromosomal rearrangements III. Cytogenetic observations on two paracentric inversions. Chromosoma 83, 419-429 (1981)
29. Poorman, P. A., M. J. Moses, L. B. Russell \& N. L. A. CACHEIRO: Synaptonemal complex analysis of mouse chromosomal arrangements I. Cytogenetic observations on a tandem duplication. Chromosoma $81,507-518$ (1981)

30. Rasmussen, S. W. \& P. B. Holm: Human meiosis II. Chromosome pairing and recombination nodules in human spermatocytes. Carlsberg Res. Commun. 43, 275-327 (1978)

31. Rouyer, F., M. C. Simmler, C. Johnsson, G. Vergnaud, H. J. CoOke \& J. Weissenbach: A gradient of sex linkage in the pseudoautosomal region of the human sex chromosomes. Nature 319, 291-295 (1986)

32. Schleiermacher. E \& W. SChmidt: Changes of the synaptonemal complex at the end of pachytene. Humangenetik 19, 235-245 (1973)

33. Searle, A. G.. R. J Berry \& C. V. Beechey: Cytogenetic radiosensitivity and chiasma frequency in wild-living male mice. Mutation Res. 9, $137-140$ (1970)

34. SPEED, R. M.: The effects of ageing on the meiotic chromosomes of male and female mice. Chromosoma 64, 241-254 (1977)

35. SPEED, R. M: Mejosis in the fetal mouse ovaries. Chromosoma 85, 427-437 (1982)

36. SOLARI.A. J.: The evolution of the ultrastructure of sex chromosomes (sex vesicle) during meiotic prophase in mouse spermatocytes. J. Ultrastruct. Res. 27, 289-305 (1969)

37. SOLARI, A. J.: The spatial relationship of the $X$ and $\mathrm{Y}$ chromosomes during meiotic prophase in mouse spermatocytes. Chromosoma 29, 217-236 (1970)

38. TRES, L. L.: Extensive pairing of the $X Y$ bivalent in mouse spermatocytes as visualized by wholemount electron microscopy. J. Cell Sci. 25, 1-15 (1977)

39. Wettstein, D. von. S. W. Rasmussen \& P. B. HoLM: The synaptonemal complex in genetic segregation. Ann. Rev. Gene1. 18, 331-413 (1984)

40. ZiCKLER, D.: Development of the synaptonemal complex and the "recombination nodules" during meiotic prophase in the seven bivalents of of the fungus Sordaria macrospora Auersw. Chromosoma $61,289-316$ (1977) 